\title{
SECONDARY CHARACTERISTIC CLASSES FOR THE ISOTROPIC GRASSMANNIAN
}

\author{
MALGORZATA MIKOSZ \\ Institute of Mathematics, Warsaw University of Technology \\ Pl. Politechniki 1, 00-661 Warsaw, Poland \\ E-mail: emmikosz@mp.pw.edu.pl
}

0. Introduction. Let $M$ be a $2 n$-dimensional manifold and $\omega$ be a 2 -form on $M$. The pair $(M, \omega)$ is called a symplectic manifold if $\omega$ is closed (i.e. $d \omega=0)$ and nondegenerate, [Wei]. A submanifold $L^{n}$ of $M$ is called Lagrangian if the restriction of the symplectic form to the tangent space $T_{y} L$ vanishes for each $y \in L$ and

$$
T_{y} L=\left(T_{y} L\right)^{\perp}=\left\{v \in T_{y} M: \omega(v, u)=0 \text { for every } u \in T_{y} L\right\} .
$$

A submanifold $I^{k}$ of $M$ is called isotropic if for every $y \in I^{k}$ a skew-orthogonal complement (with respect to $\omega$ ) of the tangent space to $I$ contains this tangent space, i.e. $\left(T_{y} I\right)^{\perp} \supseteq T_{y} I$. A submanifold $C^{2 n-k}$ is said to be coisotropic if for every $y \in C$ holds $\left(T_{y} C\right)^{\perp} \subseteq T_{y} C$. Note that the dimension of an isotropic submanifold is less than or equal to $n$, and the dimension of a coisotropic one is greater than or equal to $n$. A Lagrangian submanifold is an isotropic submanifold of maximal dimension or a coisotropic one of minimal dimension. These submanifolds play a significant role in symplectic geometry and they appear naturally in many branches of physics ([GS], [Ja1], [Ja2]).

A fundamental example of a symplectic manifold is the real cotangent bundle $T^{*} \mathbb{R}^{n}$ endowed with the canonical symplectic structure $\omega=d \Theta$, where 1-form $\Theta$ is the Liouville form on $T^{*} \mathbb{R}^{n}$. It is defined by $\Theta(u)=\left\langle T \pi_{R^{n}}(u), \tau_{T^{*} R^{n}}(u)\right\rangle$, where $u \in T\left(T^{*} \mathbb{R}^{n}\right)$, the mapping $T \pi_{R^{n}}$ is the tangent mapping of $\pi_{R^{n}}: T^{*} \mathbb{R}^{n} \longrightarrow \mathbb{R}^{n}$ and $\tau_{T^{*} R^{n}}: T\left(T^{*} \mathbb{R}^{n}\right) \longrightarrow$ $T^{*} \mathbb{R}^{n}$ is the tangent bundle projection. If $(p, x)$ are coordinates of the bundle $T^{*} \mathbb{R}^{n}$, i.e. $x_{1}, \ldots, x_{n}$ are coordinates of the base and $p_{1}, \ldots, p_{n}$ are coordinates of the fibre, then $\Theta$ and $\omega$ have the local Darboux form (see [Wei])

$$
\Theta=\sum_{i=1}^{n} p_{i} d x_{i}, \quad \omega=\Sigma_{i=1}^{n} d p_{i} \wedge d x_{i} .
$$

1991 Mathematics Subject Classification: Primary: 58C27; Secondary: 57R20, 57R45.

Partially supported by KBN 965 P03 9610.

The paper is in final form and no version of it will be published elsewhere. 
This paper is devoted to the study of cohomological invariants in the theory of Lagrangian, isotropic and coisotropic submanifolds. The theory of the Maslov canonical operator and the Maslov class for a Lagrangian submanifold was formulated by V. P. Maslov in 1965 and V. I. Arnol'd in 1967, [Mas], [Ar]. The theory of Maslov and Arnol'd was motivated by the important branches of mathematics and physics: asymptotic methods of the linear partial differential equations, quantum mechanics, representation theory, etc. The Maslov class belongs to the first cohomology group of a Lagrangian submanifold. Higher characteristic classes, described by D. B. Fuks [Fu], appear in higher dimensions $(4 t+1)$. All those classes have the same geometric property: They are obstruction for transversality of two Lagrangian subbundles in a symplectic bundle. J. M. Morvan and L. Niglio obtained secondary characteristic classes in the isotropic case, [MN]. Although their approach is less geometric. Our goal is to find geometric cycles in the isotropic Grassmannian which represent classes computed in [MN]. Using similar methods, as in Fuks' paper, we obtain the cycles in some of the expected dimensions. We show that they are nontrivial. This proves that the cycle in the lowest dimension agrees with a generator of the first nontrivial cohomology group in odd dimension.

The first section contains the main information about the Maslov class and the Maslov index theory for Lagrangian submanifolds. In the second section we recall the definition of higher characteristic classes for the Lagrangian Grassmannian. A similar, geometric construction is applied to define higher characteristic classes for the isotropic Grassmannian. Let $\mathcal{I}_{k}^{2 n}$ be the isotropic Grassmannian of $k$-dimensional isotropic subspaces in $2 n$-dimensional symplectic vector space. We obtain the cycles of codimensions $2 i+1$ for $i=n-k+1, n-k+2, \ldots, 2 n-k-1$. We show that for $i$ even the cycles are coorientable. They are nontrivial in cohomology of the isotropic Grassmannian for $i \leq 2 n-2 k+1$.

Acknowledgements. I would like to thank Professor Stanisław Janeczko for introducing me to the subject and for helping me in the preparation of the paper.

1. Maslov class and Maslov index for a Lagrangian submanifold. Let $L^{n}$ be a Lagrangian submanifold of the real cotangent bundle $T^{*} \mathbb{R}^{n}$ with the canonical symplectic form $\omega$. We denote by $\pi: T^{*} \mathbb{R}^{n} \longrightarrow \mathbb{R}^{n}$ the standard fibration of the cotangent bundle, this fibration is Lagrangian, i.e. its fibres are Lagrangian subspaces and $T^{*} \mathbb{R}^{n}$ is symplectic manifold. Let $\Gamma$ be the set of singular points of the projection $\pi_{\mid L}$.

If a submanifold $L^{n}$ is in a generic position in $T^{*} \mathbb{R}^{n}$, then the set $\Gamma$ has the following properties:

1) $\Gamma$ is a hypersurface in $L$, i.e. $\operatorname{dim}(\Gamma)=n-1$.

2) In general, the hypersurface $\Gamma$ is not smooth, because the rank of the projection $\pi$ can be less than $n-1$.

3) The set of singular points of $\Gamma$ has codimension at least three in $L$ and codimension at least two in $\Gamma$.

4) The smooth part of the hypersurface $\Gamma$ has the canonical orientation of the normal bundle.

For more details see [MSS], [Ar]. 
The set $\Gamma$ defines a canonical cohomology class for a generic Lagrangian submanifold, called the Maslov class.

Definition 1.1. The Maslov class for a generic $L^{n} \subset T^{*} \mathbb{R}^{n}$ is defined as the element $[\mathbf{V}]$ of the first cohomology group $H^{1}(L, \mathbb{Z})$ such that its evaluation on a onedimensional cycle $\gamma$ on $L$ is the intersection number of $\gamma$ and the hypersurface $\Gamma$. The evaluation of $[\mathbf{V}]$ on $\gamma$ is called the Maslov index of the curve $\gamma$ on the Lagrangian submanifold $L$, we denote it by $v(L, \gamma)$.

We should explain some details.

1) If the submanifold $L^{n}$ is oriented and compact, then the set $\Gamma$ defines an integer cycle (after a choice of an orientation). The class $[\mathbf{V}]$ is the Poincaré dual element to the class $[\Gamma] \in H_{n-1}(L, \mathbb{Z})$.

2) For a nongeneric case we can also define the Maslov class. It will be defined in Remark 1.3, as the pull-back of the universal class of the Lagrangian Grassmannian.

The Maslov class $[\mathbf{V}]$ is related to the singularities of the projection $\pi_{\mid L}$, it assigns to every Lagrangian submanifold $L^{n} \subset T^{*} \mathbb{R}^{n}$ the set of Maslov indices: $v\left(L, \gamma_{1}\right)$, $v\left(L, \gamma_{2}\right), \ldots, v\left(L, \gamma_{r}\right)$ which are integers. The Maslov index is the homology invariant of a closed path contained in $L$.

We can also define the Maslov class and the Maslov index with the aid of the Gauss mapping.

In the cotangent bundle $T^{*} \mathbb{R}^{n}$. We have the following identification:

$$
\left(x_{1}, \ldots, x_{n}, p_{1}, \ldots, p_{n}\right) \in T^{*} \mathbb{R}^{n} \longleftrightarrow\left(x_{1}+i p_{1}, \ldots, x_{n}+i p_{n}\right) \in \mathbb{C}^{n} .
$$

We denote by $\Lambda_{n}$ the Lagrangian Grassmannian, i.e. the set of all Lagrangian subspaces in $\mathbb{R}^{2 n}$. We can identify it with the quotient space $U(n) / O(n)$, where $U(n)$ is the unitary group, $O(n)$ the orthogonal group. We have $\operatorname{dim}\left(\Lambda_{n}\right)=\frac{n(n+1)}{2}$, [MSS].

Let $G$ be the Gauss mapping

$$
G: L \longrightarrow \Lambda_{n} ; \quad y \mapsto T_{y} L .
$$

In our case this mapping is globally defined because the cotangent bundle $T^{*} \mathbb{R}^{n}$ is trivial.

It is possible to define a characteristic class in the cohomology of the Grassmannian $\Lambda_{n}$ such that its pull-back to $L^{n}$ is the Maslov class.

Let $y \in T^{*} \mathbb{R}^{n}$ be an arbitrary point. We identify all Lagrange subspaces of $T_{y}\left(T^{*} \mathbb{R}^{n}\right)$ with elements of the Grassmannian $\Lambda_{n}$ by parallel translation. Denote by $\Psi \subset T_{y}\left(T^{*} \mathbb{R}^{n}\right)$ the tangent space to the fibre of the projection $\pi$ at the point $y . \Psi$ is independent of $y \in T^{*} \mathbb{R}^{n}$, because the projection $\pi$ is a linear fibration. Let $\Gamma^{\prime}$ be the set of all elements of $\Lambda_{n}$ which intersect $\Psi$ in a nongeneric way. The set $\Gamma^{\prime}$ forms a hypersurface in $\Lambda_{n}$, its singular part has codimension three in $\Lambda_{n}$. The smooth part of $\Gamma^{\prime}$ is coorientable, see [MSS].

Definition 1.2. The universal Maslov class of the Lagrangian Grassmannian is defined as the element $\left[\mathbf{V} \Lambda_{n}\right]$ of the first cohomology group $H^{1}\left(\Lambda_{n}, \mathbb{Z}\right) \simeq \mathbb{Z}$ such that its evaluation on a one-dimensional cycle $\gamma$ on $\Lambda_{n}$ is the intersection number of $\gamma$ and the hypersurface $\Gamma^{\prime}$. 
R e mark 1.3. The Maslov class [V] is the image of the universal class $\left[\mathbf{V} \Lambda_{n}\right]$, under the induced map $G^{*}: H^{1}\left(\Lambda_{n}, \mathbb{Z}\right) \longrightarrow H^{1}(L, \mathbb{Z})$.

Remark 1.4. When $n$ is odd, then the Lagrangian Grassmannian $\Lambda_{n}$ is an orientable manifold, [Fu], [Bor]. In this case the set $\Gamma^{\prime}$ defines an integer cycle. The class $\left[\mathbf{V} \Lambda_{n}\right] \in H^{1}\left(\Lambda_{n}, \mathbb{Z}\right)$ is the Poincaré dual element to the class $\left[\Gamma^{\prime}\right] \in H_{p}\left(\Lambda_{n}, \mathbb{Z}\right)$, where $p=\frac{(n+1) n}{2}-1$.

We give another equivalent definition of the Maslov class, see [MSS]. This definition is the simplest, but not geometric. Let $\operatorname{det}^{2}: \Lambda_{n} \longrightarrow S^{1}$ be defined by $\operatorname{det}^{2}([A])=(\operatorname{det} A)^{2}$, where the element $[A] \in U(n) / O(n)$ represents a Lagrangian subspace in $\mathbb{R}^{2 n}$; the columns of the matrix $A \in U(n)$ form an orthogonal basis at a Lagrangian subspace $[A]$. Since $\operatorname{det}^{2}(X)=1$, for $X \in O(n)$, the mapping $\operatorname{det}^{2}: \Lambda_{n} \rightarrow S^{1}$ is well defined.

Definition 1.5. The Maslov form of $L$ is defined as the image of the volume form for the circle $S^{1}$ under the mapping

$$
\Omega^{1}(L) \stackrel{G^{*}}{\longleftarrow} \Omega^{1}\left(\Lambda_{n}\right) \stackrel{\left(\operatorname{det}^{2}\right)^{*}}{\longleftarrow} \Omega^{1}\left(S^{1}\right),
$$

i.e.

$$
\mathbf{V}=\left(\operatorname{det}^{2} \circ G\right) *\left(\frac{1}{2 \pi i} \frac{d z}{z}\right)
$$

It is well known that

Remark 1.6. The Maslov class $[\mathbf{V}]$ of $L$ is the image of a generator of the group $H^{1}\left(S^{1}, \mathbb{Z}\right) \simeq \mathbb{Z}$, under $\left(\operatorname{det}^{2} \circ G\right)^{*}: H^{1}\left(S^{1}, \mathbb{Z}\right) \longrightarrow H^{1}(L, \mathbb{Z})$.

EXAMPLE 1.7. We calculate the Maslov indices and the Maslov form and class for the unit circle. We consider two-dimensional symplectic space $T^{*} \mathbb{R} \simeq \mathbb{R}^{2}$ with the canonical symplectic form $\omega$, and the canonical projection $\pi: T^{*} \mathbb{R} \longrightarrow \mathbb{R}, \quad(p, x) \mapsto x$, where $x$ is the coordinate of the base and $p$ of the fibre. Let $L=\left\{(x, p) \in \mathbb{R}^{2}: x^{2}+p^{2}=1\right\}$ be the circle with the counter-clockwise orientation. We see that the set $\Gamma=\{A=(-1,0)$, $B=(1,0)\}$ is a zero-dimensional cycle. We orient it with the canonical orientation of the normal bundle, then the points $A$ and $B$ have the sign plus. For every oriented closed curve $\gamma$ on $L$, the Maslov index $v(L, \gamma)$ is an even number and it is related to the number of rotations clockwise and counter-clockwise. We have the Gauss mapping $G: L \longrightarrow \Lambda_{1}$. It is easy to see that in our case the mapping $\operatorname{det}^{2} \circ G$ has the form $\operatorname{det}^{2}(G(z))=-z^{2}$, where $z=(x, p)=x+i p$. Grassmannian $\Lambda_{1}$ can be identified with $S^{1}$ and the volume form for the circle is $\frac{1}{2 \pi i} \frac{d z}{z}$. Then

$$
\left[\mathbf{V} \Lambda_{1}\right]=\left[\frac{1}{2 \pi i} \frac{d z}{z}\right] \in H^{1}\left(\Lambda_{1}, \mathbb{Z}\right), \quad[\mathbf{V}]=\left[\frac{d z}{\pi i z}\right] \in H^{1}(L, \mathbb{Z}) .
$$

Maslov class is defined not only for a Lagrangian submanifold, but for a pair and for a triple of Lagrangian submanifolds. In physical aspects of the Maslov class the Lagrangian submanifold must satisfy so called quantization conditions, these are homological conditions which have to be fulfilled by the atlas of the submanifold ([MSS], $[\mathrm{Ar}]$ ). 
2. Maslov classes for coisotropic and isotropic submanifolds. We denote by $\mathcal{I}_{k}^{2 n}$ the isotropic Grassmannian, i.e. the set of all $k$-dimensional isotropic subspaces in $T^{*} \mathbb{R}^{n}$. We have the following description: $\mathcal{I}_{k}^{2 n}$ is homeomorphic to the quotient space $U(n) /(O(k) \oplus U(n-k)), \operatorname{dim}\left(\mathcal{I}_{k}^{2 n}\right)=2(n-k) k+\frac{k(k+1)}{2}$ and $H^{1}\left(\mathcal{I}_{k}^{2 n}, \mathbb{Z}\right)=0$, [Mik], [Vai]. For every isotropic subspace, its skew-orthogonal complement is a coisotropic subspace, therefore the quotient space $\mathcal{I}_{k}^{2 n}$ is also the coisotropic Grassmannian.

We cannot define the Maslov class for isotropic and coisotropic submanifolds in a classical manner. The Maslov class for a Lagrangian submanifold $L$ is defined as the image of a generator of the group $H^{1}\left(\Lambda_{n}, \mathbb{Z}\right) \simeq \mathbb{Z}$ under $G^{*}: H^{1}\left(\Lambda_{n}, \mathbb{Z}\right) \longrightarrow H^{1}(L, \mathbb{Z})$, where $G$ is the Gauss mapping. The nature of the isotropic Grassmannian is different, its first cohomology group with integer coefficients is trivial, therefore an analogue of the Maslov class should be defined in a higher cohomology group.

D. B. Fuks gave a geometric description of generators of cohomology ring of Lagrangian Grassmannian $\Lambda_{n},[\mathrm{Fu}]$. He considered the subsets in $\Lambda_{n}$ consisting of those elements $Y \in \Lambda_{n}$ which have intersections with a fixed $m$-dimensional subspace $P^{m} \subset$ $\mathbb{C}^{n} \simeq T^{*} \mathbb{R}^{n}$ at least of dimension $l$ :

$$
L_{n}^{m, l}=\left\{Y \in \Lambda_{n}: \operatorname{dim}\left(P^{m} \cap Y\right) \geq l\right\} .
$$

Cohomology groups in odd dimensions with integer coefficients are generated by $\left[\mathbf{V} \Lambda_{n}\right]$, $b_{2}, b_{4}, \ldots, b_{n-1}$, where $\left[\mathbf{V} \Lambda_{n}\right]$ is the universal Maslov class described in the first part of this paper (Definition 1.2) and $b_{i} \in H^{2 i+1}\left(\Lambda_{n}, \mathbb{Z}\right)$ for $i=2,4, \ldots, n-1$, are defined by subsets $L_{n}^{n-i+1,2}$. Cohomology groups of $\Lambda_{n}$ in even dimensions are trivial.

J. M. Morvan and L. Niglio found generators for the cohomology ring of isotropic Grassmannian $\mathcal{I}_{k}^{2 n}$ using the theory of Lie algebras, [MN], [Bor]. The generators in even dimensions come from Chern and Pontryagin classes. The odd part is generated by higher Maslov classes, which are located in dimensions $4 t+1$ for certain range of $t$. Precisely, we have:

THEOREM 2.1. The cohomology $H^{*}\left(\mathcal{I}_{k}^{2 n}, \mathbb{R}\right)$ in odd dimensions are generated by the following elements (the index denotes the degree):

$$
\begin{array}{ll}
y_{\left(4\left[\frac{n-k+1}{2}\right]+1\right)}, \ldots, y_{(4 t+1)}, \ldots, y_{(2 n-1)}, & \text { if } k \text { is even and } n \text { is odd, } \\
y_{\left(4\left[\frac{n-k+1}{2}\right]+1\right)}, \ldots, y_{(4 t+1)}, \ldots, y_{(2 n-3)}, & \text { if } k \text { and } n \text { are even, } \\
y_{\left(4\left[\frac{n-k+1}{2}\right]+1\right)}, \ldots, y_{(4 t+1)}, \ldots, y_{(2 n-3)}, x_{(2 n-1)}, & \text { if } k \text { is odd }
\end{array}
$$

where the symbol [·] denotes the integral part of a real number.

We will give a geometric description of elements in odd dimensions. The construction is motivated by Fuks' paper. A connection with the theory of singularity will be explained.

We define the following subsets in isotropic Grassmannian $\mathcal{I}_{k}^{2 n}$. We take the fixed isotropic subspaces $P^{m} \subset \mathbb{C}^{n}, 1 \leq m \leq n$. We choose $P^{m}=\left\{x_{1}=\ldots=x_{n}=0\right.$, $\left.p_{1}=\ldots=p_{n-m}=0\right\}$, where $x_{1}, \ldots, x_{n}$ are coordinates of $\mathbb{R}^{n}$ and $p_{1}, \ldots, p_{n}$ are coordinates of the imaginary part. Let $\Sigma_{k}^{2 n}(l, m)$ be the set of those elements $X$ of the Grassmannian $\mathcal{I}_{k}^{2 n}$ which have intersections with the subspace $P^{m}$ at least $l$-dimensional:

$$
\Sigma_{k}^{2 n}(l, m)=\left\{X \in \mathcal{I}_{k}^{2 n}: \operatorname{dim}\left(P^{m} \cap X\right) \geq l\right\} .
$$


The set $\Sigma_{k}^{2 n}(l, m)$ is a singular manifold, with singularities contained in the set $\Sigma_{k}^{2 n}(l+1, m)$. We consider the desingularization of $\Sigma_{k}^{2 n}(l, m)$ :

$$
\widetilde{\Sigma}_{k}^{2 n}(l, m)=\left\{(X, M) \in \mathcal{I}_{k}^{2 n} \times G_{l}\left(P^{m}\right): M \subset\left(P^{m} \cap X\right)\right\} .
$$

where $G_{l}\left(P^{m}\right)$ is the Grassmannian of all $l$-dimensional linear subspaces in $P^{m}$. The map $\eta: \widetilde{\Sigma}_{k}^{2 n}(l, m) \longrightarrow \Sigma_{k}^{2 n}(l, m),(X, M) \mapsto X$ is a homeomorphism away of singularities.

LEMmA 2.2. The set $\Sigma_{k}^{2 n}(l, m)$ is a cycle: $\operatorname{codim}\left(\Sigma_{k}^{2 n}(l, m)\right)=2(n-k) l+\frac{l(2 k-l+1)}{2}-$ $l(m-l)$ and $\operatorname{dim}\left(\Sigma_{k}^{2 n}(l, m)\right)-\operatorname{dim}\left(\Sigma_{k}^{2 n}(l+1, m)\right)=2 n-k-m+l+1 \geq 2$.

Proof. We calculate the dimension of the set $\Sigma_{k}^{2 n}(l, m)$ from the following fibration:

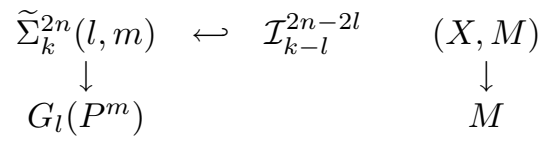

We have $l \geq 1$ and $1 \leq m \leq n$, thus $\operatorname{dim}\left(\Sigma_{k}^{2 n}(l, m)\right)-\operatorname{dim}\left(\Sigma_{k}^{2 n}(l+1, m)\right)=2 n-k-m+$ $l+1 \geq n-k+2 \geq 2$.

The singular part of $\Sigma_{k}^{2 n}(l, m)$ has codimension at least 2 , then this set defines an element in the cohomology group of $\mathcal{I}_{k}^{2 n}$ with coefficients in $\mathbb{Z}_{2}$.

Let us discuss the question of orientability. The isotropic Grassmannian is fibred in the following way:

$$
\begin{array}{ccccc}
\mathcal{I}_{k}^{2 n} & \hookleftarrow & \Lambda_{k} & Y & =\operatorname{span}_{\mathbb{R}}\left\{v_{1}, \ldots, v_{n}\right\} \\
\downarrow & & & \downarrow \\
G_{k}\left(\mathbb{C}^{n}\right) & & & Y \oplus i Y & =\operatorname{span}_{\mathbb{C}}\left\{v_{1}, \ldots, v_{n}\right\},
\end{array}
$$

where the fibre is the Lagrangian Grassmannian $\Lambda_{k}$ and the base is the classical complex Grassmannian $G_{k}\left(\mathbb{C}^{n}\right)$. The Grassmannian $G_{k}\left(\mathbb{C}^{n}\right)$ is orientable and simply connected, then $\mathcal{I}_{k}^{2 n}$ is orientable if and only if $\Lambda_{k}$ is orientable. The Grassmannian $\Lambda_{k}$ is orientable for $k$ odd (Remark 1.4). For simplicity, we assume that $k$ is odd, then the cycle $\Sigma_{k}^{2 n}(l, m)$ defines an element in cohomology with integer coefficients if and only if it is orientable.

LEMMA 2.3. For $k$ odd, $l$ and $m$ even the cycle $\Sigma_{k}^{2 n}(l, m)$ is orientable.

Proof. Using the fibration (1) we see that necessary condition of orientability of $\Sigma_{k}^{2 n}(l, m)$ is orientability of $\mathcal{I}_{k-l}^{2 n-2 l}$, then $l$ has to be even. As in Fuks' paper, the fibration is orientable and the base $G_{l}\left(P^{m}\right)$ is orientable if and only if $m$ is even.

We conclude that the cycles $\Sigma_{k}^{2 n}(l, m)$ for $k$ odd, $l$ and $m$ even, define elements in cohomology with integer coefficients. We concentrate on the case $l=2$. Then $\left[\Sigma_{k}^{2 n}(2, m)\right]$ belongs to $H^{p}\left(\mathcal{I}_{k}^{2 n}, \mathbb{Z}\right)$, where

$p=2(n-k) l+\frac{l(2 k-l+1)}{2}-l(m-l)=4(n-k)+2 k-1-2(m-2)=4 n-2 k-2 m+3$.

For $k$ even, we apply the following construction. We embed the Grassmannian $\mathcal{I}_{k}^{2 n}$ in $\mathcal{I}_{k+1}^{2 n+2}$ :

$$
i_{k}: \mathcal{I}_{k}^{2 n} \hookrightarrow \mathcal{I}_{k+1}^{2 n+2}, \quad Y \mapsto Y \oplus \mathbb{R} \oplus i\{0\}
$$

then

$$
i_{k}^{-1}\left(\Sigma_{k+1}^{2 n+2}(2, m+1)\right) \cap \mathcal{I}_{k}^{2 n}=\Sigma_{k}^{2 n}(2, m) .
$$


The Grassmannian $\mathcal{I}_{k+1}^{2 n+2}$ is orientable. For $m+1$ even, the cycle $\Sigma_{k+1}^{2 n+2}(2, m+1)$ is orientable and coorientable. Then the cycle $\Sigma_{k}^{2 n}(2, m)$ is coorientable and it also defines an element in cohomology with integer coefficients: $\left[\Sigma_{k}^{2 n}(2, m)\right] \in H^{p}\left(\mathcal{I}_{k}^{2 n}, \mathbb{Z}\right)$, where $p=4 n-2 k-2 m+3$.

Corollary 2.4.

\begin{tabular}{|c|c|c|c|}
\hline & $m$ even & $m$ odd & $\mathcal{I}_{k}^{2 n}$ \\
\hline$k$ even & $\Sigma_{k}^{2 n}(2, m)$ not coorientable & $\Sigma_{k}^{2 n}(2, m)$ coorientable & unorientable \\
\hline$k$ odd & $\Sigma_{k}^{2 n}(2, m)$ coorientable & $\Sigma_{k}^{2 n}(2, m)$ not coorientable & orientable \\
\hline
\end{tabular}

Using formula (2), we will show that the elements

$$
\left[\Sigma_{k}^{2 n}(2, m)\right] \in H^{p}\left(\mathcal{I}_{k}^{2 n}, \mathbb{Z}\right)
$$

are nontrivial for $k \leq m \leq n$ and $k+m \equiv 1(\bmod 2)$, where $p=4 n-2 m-2 k+3$. We consider the sequence of embeddings of isotropic Grassmannians:

$$
\mathcal{I}_{2}^{2(n-k+2)} \hookrightarrow \ldots \hookrightarrow \mathcal{I}_{k-1}^{2 n-2} \hookrightarrow \mathcal{I}_{k}^{2 n} .
$$

Every embedding is defined as before:

$$
i_{k}: \mathcal{I}_{k-1}^{2 n-2} \hookrightarrow \mathcal{I}_{k}^{2 n}, \quad X \mapsto X \oplus \mathbb{R} \oplus i\{0\} .
$$

Using formula (2), after suitable choice of orientation, we obtain:

$$
i_{k}^{*}\left(\left[\Sigma_{k}^{2 n}(2, m)\right]\right)=\left[\Sigma_{k-1}^{2 n-2}(2, m-1)\right] \in H^{p}\left(\mathcal{I}_{k-1}^{2 n-2}, \mathbb{Z}\right),
$$

where $4 n-2 k-2 m+3$. From here, for $m \geq k$, we have the sequence of classes:

$$
\left[\Sigma_{k}^{2 n}(2, m)\right] \mapsto\left[\Sigma_{k-1}^{2 n-2}(2, m-1)\right] \mapsto \ldots \mapsto\left[\Sigma_{2}^{2(n-k+2)}(2, m-k+2)\right],
$$

where $\Sigma_{k^{\prime}}^{2 n^{\prime}}\left(2, m^{\prime}\right) \subset \mathcal{I}_{k^{\prime}}^{2 n^{\prime}}$. At the end we have the class generated by the set

$$
\Sigma_{2}^{2(n-k+2)}(2, m-k+2) \subset \mathcal{I}_{2}^{2(n-k+2)} .
$$

Let $n^{\prime}=n-k+2$ and $m^{\prime}=m-k+2$. The codimension of $\Sigma_{2}^{2 n^{\prime}}\left(2, m^{\prime}\right)$ is $4 n^{\prime}-2 m^{\prime}-1$. We find a complementary cycle $\Theta_{2}^{2 n^{\prime}}\left(2, m^{\prime}\right) \subset \mathcal{I}_{2}^{2 n^{\prime}}$ of dimension $4 n^{\prime}-2 m^{\prime}-1$ such that the intersection of the cycle $\Theta_{2}^{2 n^{\prime}}\left(2, m^{\prime}\right)$ with $\Sigma_{2}^{2 n^{\prime}}\left(2, m^{\prime}\right)$ is a point. Let

$$
V=\left\{p_{n^{\prime}-m^{\prime}+3}=\ldots=p_{n^{\prime}}=0\right\}, \quad \operatorname{dim}(V)=2 n^{\prime}-m^{\prime}+2,
$$

be a coisotropic subspace. We recall that $P^{m^{\prime}}=\left\{x_{1}=\ldots=x_{n^{\prime}}=0, p_{1}=\ldots=p_{n^{\prime}-m^{\prime}}=0\right\}$ and $\Sigma_{2}^{2 n^{\prime}}\left(2, m^{\prime}\right)=\left\{X \in \mathcal{I}_{2}^{2 n^{\prime}}: X \subset P^{m^{\prime}}\right\}$. The complementary cycle is defined to be $\Theta_{2}^{2 n^{\prime}}\left(2, m^{\prime}\right)=\left\{X \in \mathcal{I}_{2}^{2 n^{\prime}}: X \subset V\right\}$.

Lemma 2.5. The set $\Theta_{2}^{2 n^{\prime}}\left(2, m^{\prime}\right) \subset \mathcal{I}_{2}^{2 n^{\prime}}$ is an orientable cycle of dimension $4 n^{\prime}-$ $2 m^{\prime}+1$.

Proof. 1) First we calculate the dimension. Let us apply the symplectic reduction $V \rightarrow V / V^{\perp} \simeq \mathbb{C}^{n^{\prime}-m^{\prime}+2}$, where $V^{\perp}=\operatorname{span}_{\mathbb{R}}\left\{\frac{\partial}{\partial x_{n^{\prime}-m^{\prime}+3}}, \ldots, \frac{\partial}{\partial x_{n^{\prime}}}\right\}$ is the skew-orthogonal complement to $V$ in $\mathbb{C}^{n^{\prime}}$. The generic isotropic plane in $V$ projects to an isotropic plane in $\mathbb{C}^{n^{\prime}-m^{\prime}+2}$. Thus we have a fibration $U \rightarrow \mathcal{I}_{2}^{2\left(n^{\prime}-m^{\prime}+2\right)}$, where $U \subset \Theta_{2}^{2 n^{\prime}}\left(2, m^{\prime}\right)$ is the 
set of planes which intersect $V^{\perp}$ trivially. The set $U$ is smooth. The fibre is isomorphic with $\operatorname{Hom}\left(\mathbb{R}^{2}, V^{\perp}\right)$. We calculate that

$$
\begin{aligned}
\operatorname{dim}\left(\Theta_{2}^{2 n^{\prime}}\left(2, m^{\prime}\right)\right)=\operatorname{dim}\left(\mathcal{I}_{2}^{2\left(n^{\prime}-m^{\prime}+2\right)}\right)+\operatorname{dim}\left(\operatorname{Hom}\left(\mathbb{R}^{2}, V^{\perp}\right)\right) & \\
= & 4\left(n^{\prime}-m^{\prime}\right)+3+2\left(m^{\prime}-2\right)=4 n^{\prime}-2 m^{\prime}-1 .
\end{aligned}
$$

2) We calculate the dimension of complement of $U$ in $\Theta_{2}^{2 n^{\prime}}\left(2, m^{\prime}\right)$. The generic plane from $\Theta_{2}^{2 n^{\prime}}\left(2, m^{\prime}\right) \backslash U$ projects onto a line in $\mathbb{C}^{n^{\prime}-m^{\prime}+2}$ and onto a plane in $V^{\perp}$. Its position is determined by a line in $\mathbb{C}^{n^{\prime}-m^{\prime}+2}$, a plane in $V^{\perp}$ and a map from the plane to the line. We calculate that

$$
\begin{aligned}
\operatorname{dim}\left(\Theta_{2}^{2 n^{\prime}}\left(2, m^{\prime}\right) \backslash U\right)=\operatorname{dim}\left(\mathbb{R P}^{2\left(n^{\prime}-m^{\prime}+2\right)-1}\right)+\operatorname{dim}\left(G_{2} \mathbb{R}^{\left(m^{\prime}-2\right)}\right) & \\
& +\operatorname{dim}\left(\operatorname{Hom}\left(\mathbb{R}^{2}, \mathbb{R}\right)\right)=2 n^{\prime}-3 .
\end{aligned}
$$

We check that $\operatorname{codim}\left(\Theta_{2}^{2 n^{\prime}}\left(2, m^{\prime}\right) \backslash U\right) \geq 2$. Clearly $\left(4 n^{\prime}-2 m^{\prime}-1\right)-\left(2 n^{\prime}-3\right)=2 n^{\prime}-$ $2 m^{\prime}+2 \geq 2$.

3) We show the orientability of the cycle $\Theta_{2}^{2 n^{\prime}}\left(2, m^{\prime}\right)$. We considered the vector bundle

$$
\operatorname{Hom}\left(\mathbb{R}^{2}, V^{\perp}\right) \hookrightarrow U \rightarrow \mathcal{I}_{2}^{2\left(n^{\prime}-m^{\prime}+2\right)} .
$$

The base is unorientable and the bundle is unorientable too. We note that the first homotopy group of $\mathcal{I}_{2}^{2\left(n^{\prime}-m^{\prime}+2\right)}$ equals $\mathbb{Z}_{2}$, [MSS]. Going around the only nontrivial loop, we change the orientation of $\mathbb{R}^{2}$ in $\operatorname{Hom}\left(\mathbb{R}^{2}, V^{\perp}\right)$. Since $\operatorname{dim}\left(V^{\perp}\right)=m^{\prime}-2$ is odd (because $k=2$ ), it changes the orientation of $\operatorname{Hom}\left(\mathbb{R}^{2}, V^{\perp}\right)$.

We clearly see that the intersection of the cycles $\Theta_{2}^{2 n^{\prime}}\left(2, m^{\prime}\right)$ and $\Sigma_{2}^{2 n^{\prime}}\left(2, m^{\prime}\right)$ is a single point $X=\operatorname{span}_{\mathbb{R}}\left\{\frac{\partial}{\partial p_{n^{\prime}-m^{\prime}+1}}, \frac{\partial}{\partial p_{n^{\prime}-m^{\prime}+2}}\right\} \in \mathcal{I}_{2}^{2 n^{\prime}}$. Thus $\left[\Sigma_{2}^{2 n^{\prime}}\left(2, m^{\prime}\right)\right] \in H^{*}\left(\mathcal{I}_{2}^{2 n^{\prime}}, \mathbb{Z}\right)$ is nontrivial, its evaluation on $\left[\Theta_{2}^{2 n^{\prime}}\left(2, m^{\prime}\right)\right] \in H_{*}\left(\mathcal{I}_{2}^{2 n^{\prime}}, \mathbb{Z}\right)$ equals one.

Let us summarize our results in the following corollary.

Corollary 2.6. We defined the cycles $\Sigma_{k}^{2 n}(l, m) \subset \mathcal{I}_{k}^{2 n}$ of codimensions $2 i+1$ for $i=n-k+1, n-k+2, \ldots, 2 n-k-1$. For $i$ even the cycles are coorientable. They are nontrivial in cohomology of the isotropic Grassmannian for $i \leq 2 n-2 k+1$.

We explain the relations between the obtained classes and the properties of isotropic projections. Let $M^{2 n}$ be a symplectic manifold which is fibred over a smooth base $B$, $R: M \rightarrow B$. Let the fibres of the projection $R$ be $m$-dimensional isotropic submanifolds in $M$, then we call $R$ a $m$-dimensional isotropic fibration.

ExAmple 2.7. Let $M=T^{*} \mathbb{R}^{n}$. Denote by $R_{m}$ the linear fibration:

$$
R_{m}: T^{*} \mathbb{R}^{n} \rightarrow \mathbb{R}^{2 n-m} \quad\left(x_{1}, \ldots, x_{n}, p_{1}, \ldots, p_{n}\right) \mapsto\left(x_{1}, \ldots, x_{n}, p_{1}, \ldots, p_{n-m}\right),
$$

where $0 \leq m \leq n$. The fibres are $m$-dimensional isotropic affine subspaces.

Let $I^{k}$ be a $k$-dimensional isotropic submanifold in $M$. We restrict $R$ to the submanifold $I, R_{\mid I}: I \rightarrow B$. We define a singular set $\sigma_{I}(2, m)$ of points in $I$, where the tangent mapping $T_{R_{\mid I}}$ has the rank at most $k-2$.

We consider $M^{2 n}=T^{*} \mathbb{R}^{n}$ and the fibration $R_{m}$ of $M$, Example 2.7. As in Lagrangian case, we have the Gauss mapping:

$$
F: I^{k} \rightarrow \mathcal{I}_{k}^{2 n}, \quad y \mapsto T_{y} I
$$


where the tangent spaces are identified with elements of the Grassmannian $\mathcal{I}_{k}^{2 n}$ by the parallel translation. We clearly have:

Proposition 2.8. The singular set $\sigma_{I}(2, m) \subset I$ is the inverse image of $\Sigma_{k}^{2 n}(2, m)$ under the Gauss mapping $F$.

The stratification of $\mathcal{I}_{k}^{2 n}$ is given by the dimension of intersection with the fixed isotropic subspace $P^{m} \subset I$. An isotropic submanifold in $M$ is generic if the Gauss mapping $F$ is transversal to the strata in $\mathcal{I}_{k}^{2 n}$.

Remark 2.9 .

1) If the Gauss mapping $F$ is transversal to the strata of the cycle $\Sigma_{k}^{2 n}(2, m)$, (i.e. the submanifold $I \subset M$ is in the generic position), then the set $\sigma_{I}(2, m)$ is a cycle in $I$, moreover $\left[\sigma_{I}(2, m)\right]=F^{*}\left(\left[\Sigma_{k}^{2 n}(2, m)\right]\right) \in H^{*}(I, \mathbb{Z})$.

2) The classes $\left[\sigma_{I}(2, m)\right]$ are independent of the choice of the linear isotropic fibration in $T^{*} \mathbb{R}^{n} \rightarrow \mathbb{R}^{2 n-m}$.

Remark 2.10. If the projection $T^{*} \mathbb{R}^{n} \rightarrow \mathbb{R}^{n},(p, x) \mapsto x$, restricted to the isotropic submanifold $I$ is nondegenerate, then all these sets $\Sigma_{k}^{2 n}(2, m)$ are empty. Thus the classes $\left[\Sigma_{k}^{2 n}(2, m)\right] \in H^{*}\left(\mathcal{I}_{k}^{2 n}, \mathbb{Z}\right)$ can be treated, as the cohomological obstructions on nondegeneracy of the Lagrangian projection.

We are going to treat the remaining geometric characteristic classes for the isotropic Grassmannian in the forthcoming papers.

\section{References}

[Ar] V. I. Arnol'd, On a characteristic class entering in the quantization conditions (in Russian), Funktsional. Anal. i Prilozhen. 1 (1967), 1-14.

[Bor] A. Borel, Sur la cohomologie des espaces fibrés principaux et des espaces homogènes de groupe de Lie compacts, Ann. of Math. (2) 57 (1953), 115-207.

[Fu] D. B. Fuks, Maslov-Arnol'd characteristic classes (in Russian), Dokl. Akad. Nauk SSSR 178 (1968), 303-306; English transl.: Soviet Math. Dokl. 9 (1968), 96-99.

[GS] V. Guillemin, S. Sternberg, Symplectic Techniques in Physics, Cambridge Univ. Press, Cambridge, 1984.

[Ja1] S. Janeczko, On isotropic submanifolds and evolution of quasicaustics, Pacific J. Math. 158 (1993), 317-334.

[Ja2] S. Janeczko, Coisotropic varieties and their generating families, Ann. Inst. H. Poincaré Phys. Théor. 56 (1992), 429-441.

[Mas] V. P. Maslov, Theory of Perturbation and Asymptotic Methods (in Russian), Izdat. Moskovskogo Gos. Univ., Moscow, 1965.

[Mik] M. Mikosz, On classification of the linear Lagrangian and isotropic subspaces, Demonstratio Math. 30 (1997), 437-450.

[MSS] A. S. Mishchenko, V. E. Shatalov, B. Yu. Sternin, Lagrangian Manifolds and the Method of the Canonical Operator (in Russian), Nauka, Moscow, 1978. 
[MN] J. M. Morvan, L. Niglio, Isotropic characteristic classes, Compositio Math. 91 (1994), 67-89.

[Vai] M. I. Vaisman, Symplectic Geometry and Secondary Characteristic Classes, Progr. Math. 72, Birkhäuser, Boston, 1987.

[Wei] A. Weinstein, Lectures on Symplectic Manifolds, CBMS Regional Conf. Ser. in Math. 29, Amer. Math. Soc., Providence, 1977; corrected reprint, 1979. 